\title{
Stem cell impregnated nanofiber stent sleeve for on-stent production and intravascular delivery of paracrine factors
}

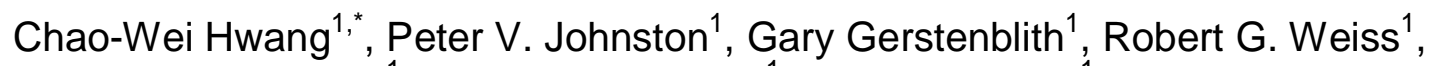
Gordon F. Tomaselli ${ }^{1}$, Virginia E. Bogdan ${ }^{1}$, Asmi Panigrahi ${ }^{1}$, Aleksandra Leszczynska ${ }^{1}$, Zhiyong $\mathrm{Xia}^{2}$

1. Division of Cardiology, Johns Hopkins University School of Medicine, Baltimore, Maryland.

2. Research and Exploratory Development Department, Johns Hopkins University Applied Physics Laboratory, Laurel, Maryland.

\section{Keywords:}

Stem cell; nanofiber; coronary artery disease; peripheral artery disease; paracrine factor

Running title:

Stem cell impregnated nanofiber stent sleeve

\section{* Corresponding author:}

Chao-Wei Hwang, M.D., Ph.D.

Division of Cardiology,

Johns Hopkins University School of Medicine

720 Rutland Avenue, Ross 1165

Baltimore, Maryland 21205

E-mail: chwang7@jhmi.edu 


\section{ABSTRACT}

Stem cell therapies for atherosclerotic diseases are promising, but benefits remain modest with present cell delivery devices in part due to cell washout and immune attack. Many stem cell effects are believed mediated by paracrine factors (PFs) secreted by the stem cells which effect tissue repair via activation and enhancement of intrinsic host repair mechanisms We therefore sought to create an "intravascular paracrine factor factory" by harnessing stem cells on a stent using a nanofiber (NF) stent sleeve, and thus providing a sheltered milieu for cells to continuously produce PFs on-stent. The NF sleeve acts as a substrate on which stem cells grow, and as a semipermeable barrier that protects cells from washout and host immune response while allowing free outward passage of PFs. NF stent sleeves were created by covering stents with electrospun poly-lactic-co-glycolic acid nanofibers and were then uniformly coated with mesenchymal stem cells (MSCs). NF sleeves blocked cell passage but did not hamper MSC attachment or proliferation, and did not alter MSC morphology or surface markers. NF sleeve MSCs continued to secrete PFs that were biologically active and successfully induced tubulogenesis in human endothelial cells. NF stent sleeves seeded with allogeneic MSCs implanted in pigs remained patent at 7 days without thrombotic occlusion or immune rejection. Our results demonstrate the feasibility of creating an intravascular PF factory using a stem cell impregnated NF stent sleeve, and pave the way for animal studies to assess the efficacy of local PF production to treat ischemic artery disease. 


\section{Introduction}

The final common pathway for most forms of coronary and peripheral artery diseases is damage to downstream tissue. Several clinical trials performed to investigate the utility of intravascular infusion and intramuscular injection of stem cells to repair muscle damage[1-5] demonstrated only modest beneficial effects[2, 6-9]. Current methods of cell delivery are thought to be limited by rapid loss of viable cells due to washout into the bloodstream, host immune response, and exposure to an inflamed local environment[10-13]. However, growing evidence suggests that many of the beneficial effects of stem cells are actually mediated by paracrine processes. It is thought that in addition to tissue repair via direct engraftment, stem cells release paracrine factors (PFs) that activate host cells, promote homing of other cells to the site of injury and thus enhance the host's intrinsic repair mechanisms[14-20].

If the reparative capacity of stem cells derives to a significant extent from their secreted PFs, then prolonging exposure of the damaged tissues to these PFs would augment the reparative response. In this work, we sought to create an intravascular "paracrine factor factory" to locally produce and deliver PFs by harnessing stem cells within a sheltered environment attached to a stent. Using an electrospinning technique, we constructed a nanofiber (NF) stent sleeve which we impregnated with a large number of stem cells. The mesh-like NF sleeve acts as a substrate on which the stem cells grow, and as a protective semi-permeable barrier that prevents washout of the stem cells into the bloodstream, excludes host immune cells, and at the same time, allows free passage of PFs, nutrients and wastes as well as the host's signaling cytokines (Figure 1). With the stem cells protected, the NF stent sleeve behaves as an 
intravascular factory that produces and releases PFs directly into the circulation. Inflammatory cytokines released by ischemic tissue[21], such as IL-1, IL-8, TNF- $\alpha$, or TGF- $\beta$, could be sensed by NF sleeve stem cells, and PF production and release therefore tailored according to the evolving needs of the healing tissue.

In this study, we demonstrate the technical feasibility of creating an intravascular PF factory using a stem cell impregnated NF stent sleeve. We show that NF sleeves prevent cell passage but do not hamper MSC attachment or proliferation, and do not alter stem cell morphology or stem cell surface markers. The NF stent sleeve stem cells continue to produce relevant PFs, and these PFs have biological functionality. Finally, we successfully implanted a NF stent sleeve impregnated with allogeneic MSCs in the pig umbilical artery for 7 days and demonstrate biocompatibility and absence of immune rejection. These data pave the way for larger animal studies to assess the effectiveness of a local paracrine-based approach in reversing some of the consequences of coronary and peripheral artery diseases. 


\section{Materials \& Methods}

\section{Nanofiber Stent Sleeve}

Fabrication: Nanofiber stent sleeves are electrospun onto the external surfaces of nonmedicated cobalt chromium stents (Medtronic, Minneapolis, MN). Poly (Llactide/glycolide) acid (PLGA) co-polymer (PLG 8531; PURAC, Lincolnshire, IL) was dissolved in a mixture of dichloromethane and methanol (7:3 vol:vol). The stents were balloon-expanded to their nominal diameters and removed from the balloon. The copolymer was then electrospun onto the stent at $12 \mathrm{kV}$ (Gamma High Voltage Research Model ES30P-5W/DAM, Ormond Beach, FL) using a syringe pump (NE-300 Infusion Pump; New Era Pump Systems, Farmingdale, NY) with a flow rate of $3.5 \mathrm{~mL} / \mathrm{hr}$ via an $22 \mathrm{G} \times 1.5$ inch blunt-tip needle at a distance of $9 \mathrm{~cm}$ from the collecting target for 25 minutes. To produce a uniform NF sleeve thickness, the stent was continuously rotated along its longitudinal axis during electrospinning at 130 revolutions per minute. The completed electrospun NF stents were sterilized in a solution of $70 \%$ ethanol for 12 hours. Similar electrospinning techniques are described in the literature to fabricate covered stents[22, 23] and vascular grafts[24].

Pore Size Characterization: NF sleeve morphology was imaged using a Hitachi S4700 scanning electron microscope (SEM) at baseline before and after crimping onto a $2.5 \mathrm{x}$ $12 \mathrm{~mm}$ angioplasty balloon (Medtronic, Minneapolis, MN) using a radial compression stent crimper (Model RMC, Blockwise Engineering, Phoenix, AZ) and re-expanding to nominal diameter $(n=3)$. Pore diameters were measured using image processing tools in MATLAB (Mathworks, Natick, MA). Specifically, pixel intensity thresholds were used to define pores on the SEM images. Since pores are randomly oriented across the NF 
sleeve and are of different shapes and sizes, at least 500 individual samples of intensity profiles were taken across each SEM image, and the lengths of continuous segments of pixel intensities below the threshold defined as a pore were quantified, averaged, and taken to be the average pore diameter.

Cell Permeability: A NF stent sleeve was expanded to join two separate segments of tubing (Tygon S-50HL, 3/32" inner diameter, Cole-Palmer). A gap of $0.5 \mathrm{~cm}$ was left between the two segments, so that part of the nanofiber stent sleeve remained not covered by the tubing. The construct was placed in a media bath, and a solution of $10^{6}$ mesenchymal stem cells was placed into the tubing, filling the stent sleeve lumen. Outward stem cell transit from the NF stent sleeve lumen to the media bath through the exposed portion of the stent sleeve was quantified after 6 hours and 60 hours in culture.

\section{$\underline{\text { NF Stent Sleeve Impregnation, Overcoating, Cell Coverage and Cell Viability }}$}

NF Sleeve Impregnation and Overcoating: Pig mesenchymal stem cells (pMSCs) were isolated and expanded from bone marrow biopsies of Yorkshire farm pigs using methods detailed previously[25, 26]. For NF impregnation, pMSCs at passages 4-6 were harvested using $0.25 \%$ Trypsin-EDTA (Gibco), and re-suspended in culture medium at a concentration of $3 \times 10^{6} \mathrm{pMSCs} / \mathrm{mL}$. NF stent sleeves were then placed in the pMSC suspension in a $1.5 \mathrm{~mL}$ Eppendorf tube which was gently rotated for 30 minutes at $37^{\circ} \mathrm{C}$ to ensure uniform cell adhesion. Excess non-adherent stem cells were washed off with fresh medium. The cell-impregnated NF stent sleeves were then incubated in cell culture medium (Alpha-MEM, Life Technologies) supplemented with fetal bovine serum for at least an additional 24 hours prior to use. Cell-impregnated NF 
stent sleeves can be overcoated with an additional NF sleeve layer outside the cell layer. Cell-impregnated NF stent sleeves for overcoating were cultured for 7 days prior to electrospinning under sterile conditions with another layer of NF for $12 \mathrm{~min}$, and were cultured after overcoating for 3 to 6 days prior to use.

Cell Coverage and Viability: Cell coverage and stem cell viability on the NF stent sleeve were evaluated on days 1 and 8 after cell impregnation using a Calcein-AM/Ethidium homodimer assay (LIVE/DEAD Viability Assay, Life Technologies). Cell-impregnated NF stent sleeves were incubated in $2 \mu \mathrm{M}$ Calcein-AM and $4 \mu \mathrm{M}$ Ethidium homodimer solution for 30 minutes. The NF stent sleeves were briefly washed in phosphate buffered saline and then imaged using confocal fluorescence microscopy (Zeiss AxioObserver with 710NLO-Meta confocal module) to assess NF sleeve surface cell coverage and any cell infiltration into the sleeve matrix.

\section{Flow Cytometry}

To confirm that the NF sleeve does not induce differentiation of impregnated cells, pMSCs were collected and analyzed for expression of MSC-specific surface markers by flow cytometry seven days after incorporation in the NF sleeve. Cells were analyzed for CD90, CD44, and CD34 expressions using commercially available antibodies (BD Bioscience) and samples were analyzed using FACS LSR-II (BD Bioscience). Data were gated, analyzed and compensated using FlowJo software (Tree Star, Ashland OR). 7-Aminoactinomycin-D (7-AAD) stained nonviable cells and cell aggregates were excluded during the analysis. To reliably distinguish positive and negative staining populations, standard isotype controls (BD Bioscience) were used as negative controls. 


\section{Paracrine Factor Production and In Vitro Tubulogenesis Assays}

Paracrine Factor Production: Human (hMSCs) were obtained from Lonza (Walkersville, Maryland) and expanded using standard methods[27]. hMSCs were used for the paracrine factor production assays because ELISA kits available commercially for hMSCs have a superior dynamic range and better reproducibility than do those available for pMSCs. The hMSCs were embedded into $1 \times 1 \mathrm{~cm}^{2} \mathrm{NF}$ stent sleeve patches by incubating the sleeves in a $0.5 \mathrm{~mL}$ solution of $2 \times 10^{5} \mathrm{hMSC}$ (passage number 6) for 90 minutes, and then washing off the excess unattached hMSCs. Conditioned media was collected 1, 3, and 7 days after stem cell seeding. Secreted vascular endothelial growth factor (VEGF) and hepatocyte growth factor (HGF) were analyzed using enzyme-linked immunosorbent assays (Quantikine ELISA kits, R\&D Systems; Minneapolis, MN).

In Vitro Tubulogenesis: For the Human Umbilical Vein Endothelial Cell (HUVEC) tubule formation assay, 6-well plates were coated with 250 microliters of Geltrex (Invitrogen) and seeded with $1.8 \times 10^{5}$ HUVECs suspended in $2.5 \mathrm{~mL}$ of Medium 200PRF (Invitrogen). $1 \times 1 \mathrm{~cm}^{2} \mathrm{NF}$ sleeves $(n=4)$ were seeded with $\mathrm{hMSCs}$ and incubated for 7 days before being placed on top of the HUVECs in each well. NF sleeves with a NF overcoat $(n=3)$ were incubated for an additional 3 days after the overcoating process before being placed into the HUVEC wells. To be certain that the sleeves themselves could not induce tubulogenesis, negative controls consisted of a $1 \times 1 \mathrm{~cm}^{2} \mathrm{NF}$ sleeve without hMSCs placed on top of the HUVECs. Positive controls consisted of a solution of low serum growth supplement (LSGS, Life Technologies) containing PFs designed to 
activate HUVEC tubulogenesis. Images of the tubule network were obtained 18 hours after placing the NF sleeves in the HUVEC wells. To quantify tubule formation, five microscopy images were taken at pre-defined positions in the HUVEC wells. The total pixel length of the tubules was measured in each image using ImageJ software $(\mathrm{NIH}$, Bethesda, MD), and averaged over the five images.

\section{Endovascular Implantation in Swine}

NF sleeves were electrospun onto balloon-expanded non-medicated cobalt chromium bare metal stents (Medtronic, Minneapolis, MN), crimped back down onto its stent delivery balloon (Medtronic, Minneapolis, MN) using a radial compression stent crimper (Model RMC, Blockwise Engineering, Phoenix, AZ), and seeded with allogeneic pMSCs as detailed above. The stent was delivered over a $180 \mathrm{~cm}$ Luge wire (Boston Scientific, Natick, MA) via an 8-French Judkins Right 4 (JR4) Vista Brite Tip guiding catheter (Cordis, Bridgewater, $\mathrm{NJ}$ ) to the mid-segment of the pig left umbilical artery where it was deployed at $9 \mathrm{~atm}$ and post-dilated to 12 atm to ensure vessel wall apposition. The pig was treated with aspirin $324 \mathrm{mg}$, clopidogrel $600 \mathrm{mg}$, and intravenous unfractionated heparin $5000 \mathrm{U}$ pre-procedure, and received daily aspirin $81 \mathrm{mg}$ and clopidogrel $75 \mathrm{mg}$ post-procedure. The angiographic appearance of the umbilical artery was recorded using manual injection of iodinated contrast (Omnipaque, GE Healthcare, Princeton, NJ) immediately pre-procedure, during stent inflation, and at 60 minutes and 7 days postimplantation. All animal experiments were performed under protocol SW14M120 approved by the Johns Hopkins University Animal Care and Use Committee. 


\section{Results}

\section{$\underline{\text { Architecture of NF Stent Sleeves }}$}

We first show that the porosity of the NF sleeve allows the sleeve to act as an effective barrier that can prevent stem cell washout into the bloodstream, exclude host cell infiltration, and at the same time allow free passage of PFs. SEM imaging shows that the electrospun NF stent sleeves consist of randomly oriented fibers which uniformly cover the stent (Figure 2A). NF sleeve thickness averaged $80.0 \pm 17.6$ um with pore diameters of $1.02 \pm 0.30$ um and a narrow distribution in each sleeve (Figure 2B). Crimping the stent onto an angioplasty balloon followed by balloon re-expansion, as required for clinical implantation, did not significantly affect NF sleeve pore diameters, which were $0.91 \pm 0.10$ um after re-expansion. Individual nanofibers did show minor stretch artifact without structural failure after balloon re-expansion compared to baseline (Figures 2C vs. 2D). The architecture of the NF sleeve can be slightly changed by modulating electrospinning conditions. Increasing voltage from $12 \mathrm{kV}$ to $18 \mathrm{kV}$ (Figures 2C vs. $2 \mathrm{E}$ ), for instance, resulted in qualitatively more organized fiber orientations but pore diameters, $0.82 \pm 0.19 \mathrm{um}$, were not markedly different and unlikely to affect permeability to cells or to PFs.

NF stent sleeve pore diameters are an order of magnitude smaller than typical stem cell diameters of 15-25 um, and two orders of magnitude larger than typical PF molecular sizes of tens of $\mathrm{nm}$. Not surprisingly therefore, our permeability experiments show that the NF stent sleeves are impermeable to cells. $10^{6} \mathrm{MSCs}$ were placed in the lumen of NF stent sleeves and allowed to freely transit across the NF sleeve. After 6 hours and after 60 hours, no MSCs were detected outside the NF stent sleeves. Additionally, cells 
are unable to infiltrate into the NF sleeve matrix. Confocal fluorescence imaging of stem cell impregnated NF sleeves after 7 days in culture demonstrated a monolayer of cells on the curved surface of the sleeves (Figure 3) with confocal cross-sections of the NF sleeves showing no evidence of cell infiltration into the matrix of the 80 -um thick sleeves. The NF sleeve itself has no significant autofluorescence (Figures 4E-F).

\section{Stem Cell Attachment and Viability}

We next show that the NF sleeve barrier does not inhibit stem cell attachment and growth. Pig MSCs were seeded on the NF stent sleeve, and confocal fluorescence microscopy LIVE/DEAD images of the portions of the NF sleeve on the stent strut and between stent struts were obtained on day 1 and day 8 (Figures 4A-D). MSCs attached easily to the NF sleeve and proliferated readily. On day 1 after seeding, MSC coverage of the sleeve was sparse, but the cells proliferated on the sleeve and became confluent by day 8 (Figures 4A vs. 4C). MSCs maintained their healthy elongated morphologies, with excellent viability. Control sleeves had no cells (Figures 4E-F) and no autofluorescence. Interestingly, MSC viability was poor and seeding was sparse for those locations of the sleeve immediately over the stent struts (Figures 4B, 4D), possibly reflecting stent strut impediment of efficient nutrient and waste transfer.

Overcoating, the addition of a second layer of NF to sandwich the cells (Figure 5A), while not necessary for the NF sleeve's primary purpose as a lumen barrier, could be important if the cells were particularly immunogenic. The primary NF sleeve layer prevents immune cells from reaching the MSCs from the vessel lumen; the NF sleeve overcoat blocks cells entering through the vessel wall. Overcoating cell-impregnated 
sleeves after 7 days with an additional layer of NF resulted in an initial steep drop in the number of viable cells in the sleeve due to the electrospinning process (Figure 5B), but after 6 additional days in culture post-overcoating, confluence was restored with excellent viability (Figure $5 \mathrm{C}$ ). These data suggest that excellent transfer of nutrients, wastes and PFs through the sleeve is retained with the overcoat, and are consistent with typical pore diameters of the NF sleeve two orders of magnitude larger than typical molecular diameters.

\section{$\underline{\text { Retention of Stem Cell Characteristics }}$}

As our goal is to create an intravascular PF factory, MSC retention of their stem cell characteristics in the NF sleeve is important since differentiated cells may not be able to produce certain PFs. Flow cytometry analysis showed that pig MSCs seeded in the NF stent sleeve retain the typical cell surface markers expressed by stem cells after 7 days (Figure 6). Patterns of cell surface marker expression of CD90, CD44, and CD34 in pigs MSCs embedded within the sleeve were identical to those of pig MSCs grown in culture de novo. The materials and fibrous structure of the NF stent sleeve therefore do not induce differentiation of seeded MSCs.

\section{MSCs produce relevant PFs and induce in vitro tubulogenesis}

We show that MSCs in the NF sleeves continue to make PFs, and that these PFs are biologically active. From the library of potential PFs secreted by the MSCs, we selected vascular endothelial growth factor (VEGF) and hepatocyte growth factor (HGF) as relevant indicator PFs to track. NF sleeves seeded with hMSCs produced VEGF and 
HGF continuously over 7 days in culture (Figure 7). The average rate of paracrine factor production did not change from day 1 to day 7 . Control NF sleeves without hMSCs incubated in culture media showed trace amounts of PF, likely due to baseline noise or interference with the ELISA assay (Figure 7).

Whereas the ELISA assay demonstrated that NF sleeve stem cells produce PFs, we next performed a tubulogenesis assay to show that these PFs are biologically functional. HUVECs exposed to NF sleeves with hMSCs organized into tubules to a far greater extent than those exposed to control NF sleeves without hMSCs (Figure 8). NF sleeves with hMSCs and no overcoat induced $3.1 \pm 0.7$ fold greater tubulogenesis compared to negative control $(p<0.01)$. NF sleeves with hMSCs and an overcoat induced $3.6 \pm 0.1$ fold greater tubulogenesis compared to negative controls $(p<0.01)$. The NF sleeve overcoat did not impact the ability of the NF sleeve hMSCs to induce tubulogenesis. Interestingly, NF sleeves with hMSCs induced HUVEC tubulogenesis to a similar degree as the low serum growth supplement (LSGS) positive control. This is a remarkable result considering that the NF sleeves, unlike the LSGS medium, are not pre-loaded with PFs. The NF sleeve hMSCs therefore produced all necessary PFs to induce HUVEC tubulogenesis within the 18-hour span of the assay. Taken together, these data suggest that NF sleeve MSCs retain the ability to produce abundant levels of relevant PFs that retain biological functionality.

\section{Pig Endovascular Implantation}

We successfully implanted NF stent sleeves seeded with allogeneic pMSCs into the left umbilical artery of Yorkshire swine using standard clinical vascular guidewires and 
guiding catheters. The angiographic appearance of the left umbilical artery preimplantation (Figure 9A) was recorded, and the NF stent sleeve was deployed in the mid left umbilical artery (Figure 9B) with vessel occlusion during balloon inflation confirming stent apposition to the vessel wall. There were no peri-procedural complications, and no adverse events were observed up to 1 week after implantation. A daily regimen of aspirin $81 \mathrm{mg}$ and clopidogrel $75 \mathrm{mg}$ was tolerated without any adverse effects. The angiographic appearances of the artery at 60 minutes (Figure $9 \mathrm{C}$ ) and at 7 days (Figure 9D) after implantation were not substantially different from its appearance pre-implantation. Normal (TIMI Grade 3) blood flow was maintained. There was no evidence of thrombotic reaction, and importantly, there was no evidence of immune rejection of the allogeneic cells within the NF sleeve. 


\section{Discussion}

Paracrine signaling is increasingly believed to be one of the primary mechanisms by which stem cells exert their effects[14-16, 18, 20]. Paracrine factors secreted by stem cells, including growth factors[14, 20, 28], micro-RNAs, and exosomes[29, 30], promote intrinsic repair processes in injured tissue. For example, extracts of bone marrow cells containing only secreted PFs without cells improved cardiac ejection fraction to the same extent as did direct intramyocardial injection of bone marrow cells in a mouse model of myocardial infarction[19]. In another study, intracoronary injection of a solution of PFs secreted by MSCs improved myocardial contractility and cardiomyocyte resistance to ischemia in a swine model of myocardial infarction[20]. If PFs are central mediators in tissue repair, and if stem cells exert their effects through PFs, then a large number of stem cells sheltered in a protected intravascular environment close to the site of injury, shielded from washout and protected from the host immune response, would, in theory, be able to produce abundant, relevant, PFs that would enhance downstream tissue healing.

We developed the stem cell impregnated nanofiber sleeve in an effort to create a stent-based intravascular paracrine factor factory that can continuously produce PFs locally, in vivo, to potentiate tissue repair. The NF sleeve provides a convenient method to immobilize stem cells on an intravascular stent, and our data show that the NF sleeve is an effective barrier against cell entry and exit. Our cell proliferation and viability data show that in addition to being an effective semi-permeable barrier, the NF sleeve is a favorable substrate for stem cells. Stem cells within the NF stent sleeve proliferate robustly, retain their stem cell characteristics, and produce biologically relevant PFs. 
The NF sleeve porosity is such that PFs and host signaling cytokines easily pass, implying that stem cells on the sleeve can sense the needs of local injured tissue, and adjust their PF production and release accordingly. Stem cells are protected by the NF sleeve from washout into the bloodstream and are shielded by the sleeve from host cells. Because host immune cells are excluded, non-autologous cells (allogeneic or even xenogeneic) can be deployed with the stent sleeve. Many clinical studies have been limited to autologous cells[1, 2, 4, 7] to avoid an immune response, but the advanced age and multiple co-morbidities of elderly patients may reduce the potency of their stem cells[10, 31]. Within the environment of the NF stent sleeve, allogeneic cells from young, healthy donors could be cultured to large numbers, stored for immediate availability, and then used right at the time of injury.

Using an intravascular PF factory to locally make and locally deliver PFs has several advantages when compared to methods of delivery via traditional drug delivery vehicles such as drug-eluting stents (DES). Traditional delivery vehicles can be preloaded with only a set number of PFs and are manufactured with a limited number of pre-defined delivery profiles. The number of PFs involved in tissue healing, however, is vast, with different PFs in different combinations, concentrations, and sequences likely to be involved at different phases of healing. Successfully fine-tuning traditional drug delivery vehicles to release such a vast number of PFs at different levels fluctuating in time is not feasible. Stem cells naturally do this in vivo, and can potentially produce the entire library of PFs. Our data suggest that stem cells' natural PF production capabilities are not hampered after being harnessed in the NF stent sleeve. In addition, unlike traditional delivery vehicles, stem cells can sense host cytokines, and can 
therefore adapt their PF release based on changes in their local milieu.

To our knowledge, this is the first report of an on-stent stem cell sleeve designed for intravascular paracrine factor production and delivery. NFs are used for other purposes in the vascular setting. The use of polyurethane NF covered stents to exclude intracranial aneurysms has been described[32], and other investigators proposed using NF covered stents in saphenous vein graft interventions[33] to reduce distal embolization. The large surface area of the NF matrix can also be used as a traditional drug delivery platform, and drug-eluting NF covered stents have been used to deliver paclitaxel[34], rosuvastatin[35], aspirin[36], as well as nanoparticles containing steroids[22]. The porous nature of the NF matrix is also well suited for tissue engineering applications. Pre-shaped NF matrices are used as vascular scaffolds to create biological vascular prostheses in situ via in vivo seeding of the matrices with vascular cells[24]. The stem cell impregnated NF stent sleeve fundamentally differs from such vascular scaffolds however, as migration of host cells into the vascular scaffold is purposely encouraged, whereas such cell migration is specifically blocked in our NF stent sleeves.

Cell-coated non-NF stents are also described, but much of this field has focused on promoting re-endothelialization in the immediate vascular segment of the stent[3740]. Other cell-coated stents have not, to our knowledge, been described as intravascular PF-producing factories to potentiate healing of both local and downstream tissue. Several authors describe functionalizing stent surfaces to facilitate capture, adhesion and local growth of progenitor cells or vascular endothelial cells[38, 39, 41]. In particular, the Genous stent, a stent coated with CD34 antibodies to capture 
circulating endothelial progenitor cells, was evaluated in several human clinical trials, and shows some promise in limiting stent thrombosis[41] although effects on preventing restenosis in complex lesions are more modest[38]. It is our expectation that the concentrated presence of stem cells on our NF stent sleeve producing pro-healing PFs could have a robust effect in promoting repair of damaged tissue.

\section{Conclusion}

Many stem cell effects are paracrine-mediated; PFs secreted by stem cells potentiate endogenous repair. In this work, we describe an intravascular PF factory based on a novel stem cell impregnated NF stent sleeve which facilitates the sustained intravascular production and release of PFs by stem cells shielded from washout and host immune response. The device can be delivered and deployed in the vasculature using standard clinical techniques. Our results confirm the engineering feasibility and biological functionality of a stem cell impregnated NF stent sleeve, and pave the way for further studies to assess local intravascular PF production as a strategy to treat ischemic tissue damage. 


\section{Acknowledgements}

This study was supported by funding from The Magic That Matters Fund, the Zegar Family Foundation, the William F. Keating Endowment Award from the American College of Cardiology Foundation, and an Independent Research and Development (IRAD) grant from the Johns Hopkins University Applied Physics Laboratory. 


\section{References}

[1] Assmus B, Rolf A, Erbs S, Elsasser A, Haberbosch W, Hambrecht R, et al. Clinical outcome 2 years after intracoronary administration of bone marrow-derived progenitor cells in acute myocardial infarction. Circulation Heart failure. 2010;3:89-96.

[2] Bolli R, Chugh AR, D'Amario D, Loughran JH, Stoddard MF, Ikram S, et al. Cardiac stem cells in patients with ischaemic cardiomyopathy (SCIPIO): initial results of a randomised phase 1 trial. Lancet. 2011;378:1847-57.

[3] Hare JM, Fishman JE, Gerstenblith G, Difede Velazquez DL, Zambrano JP, Suncion VY, et al. Comparison of allogeneic vs autologous bone marrow-derived mesenchymal stem cells delivered by transendocardial injection in patients with ischemic cardiomyopathy: the POSEIDON randomized trial. JAMA : the journal of the American Medical Association. 2012;308:2369-79.

[4] Makkar RR, Smith RR, Cheng K, Malliaras K, Thomson LE, Berman D, et al. Intracoronary cardiosphere-derived cells for heart regeneration after myocardial infarction (CADUCEUS): a prospective, randomised phase 1 trial. Lancet. 2012;379:895-904.

[5] Traverse JH, Henry TD, Pepine CJ, Willerson JT, Zhao DX, Ellis SG, et al. Effect of the use and timing of bone marrow mononuclear cell delivery on left ventricular function after acute myocardial infarction: the TIME randomized trial. JAMA : the journal of the American Medical Association. 2012;308:2380-9.

[6] Heldman AW, DiFede DL, Fishman JE, Zambrano JP, Trachtenberg BH, Karantalis V, et al. Transendocardial mesenchymal stem cells and mononuclear bone marrow cells for ischemic cardiomyopathy: the TAC-HFT randomized trial. JAMA : the journal of the American Medical Association. 2014;311:62-73.

[7] Malliaras K, Makkar RR, Smith RR, Cheng K, Wu E, Bonow RO, et al. Intracoronary cardiospherederived cells after myocardial infarction: evidence of therapeutic regeneration in the final 1-year results of the CADUCEUS trial (CArdiosphere-Derived aUtologous stem CElls to reverse ventricUlar dySfunction). Journal of the American College of Cardiology. 2014;63:110-22.

[8] Suncion VY, Ghersin E, Fishman JE, Zambrano JP, Karantalis V, Mandel N, et al. Does transendocardial injection of mesenchymal stem cells improve myocardial function locally or globally?: An analysis from the Percutaneous Stem Cell Injection Delivery Effects on Neomyogenesis (POSEIDON) randomized trial. Circulation research. 2014;114:1292-301.

[9] Nowbar AN, Mielewczik M, Karavassilis M, Dehbi HM, Shun-Shin MJ, Jones S, et al. Discrepancies in autologous bone marrow stem cell trials and enhancement of ejection fraction (DAMASCENE): weighted regression and meta-analysis. Bmj. 2014;348:g2688.

[10] Dimmeler S, Leri A. Aging and disease as modifiers of efficacy of cell therapy. Circulation research. 2008;102:1319-30.

[11] Terrovitis JV, Smith RR, Marban E. Assessment and optimization of cell engraftment after transplantation into the heart. Circulation research. 2010;106:479-94.

[12] Bonios M, Terrovitis J, Chang CY, Engles JM, Higuchi T, Lautamaki R, et al. Myocardial substrate and route of administration determine acute cardiac retention and lung bio-distribution of cardiospherederived cells. Journal of nuclear cardiology : official publication of the American Society of Nuclear Cardiology. 2011;18:443-50.

[13] Huang XP, Sun Z, Miyagi Y, McDonald Kinkaid H, Zhang L, Weisel RD, et al. Differentiation of allogeneic mesenchymal stem cells induces immunogenicity and limits their long-term benefits for myocardial repair. Circulation. 2010;122:2419-29.

[14] Gnecchi M, Zhang Z, Ni A, Dzau VJ. Paracrine mechanisms in adult stem cell signaling and therapy. Circulation research. 2008;103:1204-19. 
[15] Baraniak PR, McDevitt TC. Stem cell paracrine actions and tissue regeneration. Regenerative medicine. 2010;5:121-43.

[16] Chimenti I, Smith RR, Li TS, Gerstenblith G, Messina E, Giacomello A, et al. Relative roles of direct regeneration versus paracrine effects of human cardiosphere-derived cells transplanted into infarcted mice. Circulation research. 2010;106:971-80.

[17] Gu M, Nguyen PK, Lee AS, Xu D, Hu S, Plews JR, et al. Microfluidic single-cell analysis shows that porcine induced pluripotent stem cell-derived endothelial cells improve myocardial function by paracrine activation. Circulation research. 2012;111:882-93.

[18] Li TS, Cheng K, Malliaras K, Smith RR, Zhang Y, Sun B, et al. Direct comparison of different stem cell types and subpopulations reveals superior paracrine potency and myocardial repair efficacy with cardiosphere-derived cells. Journal of the American College of Cardiology. 2012;59:942-53.

[19] Yeghiazarians Y, Zhang Y, Prasad M, Shih H, Saini SA, Takagawa J, et al. Injection of bone marrow cell extract into infarcted hearts results in functional improvement comparable to intact cell therapy. Molecular therapy : the journal of the American Society of Gene Therapy. 2009;17:1250-6.

[20] Nguyen BK, Maltais S, Perrault LP, Tanguay JF, Tardif JC, Stevens LM, et al. Improved function and myocardial repair of infarcted heart by intracoronary injection of mesenchymal stem cell-derived growth factors. Journal of cardiovascular translational research. 2010;3:547-58.

[21] Sharma HS, Das DK. Role of cytokines in myocardial ischemia and reperfusion. Mediators of inflammation. 1997;6:175-83.

[22] Oh B, Lee $\mathrm{CH}$. Advanced cardiovascular stent coated with nanofiber. Molecular pharmaceutics. 2013;10:4432-42.

[23] Wang JB, Zhou B, Gu XL, Li MH, Gu BX, Wang W, et al. Treatment of a canine carotid artery aneurysm model with a biodegradable nanofiber-covered stent: a prospective pilot study. Neurology India. 2013;61:282-7.

[24] Mrowczynski W, Mugnai D, de Valence S, Tille JC, Khabiri E, Cikirikcioglu M, et al. Porcine carotid artery replacement with biodegradable electrospun poly-e-caprolactone vascular prosthesis. Journal of vascular surgery. 2014;59:210-9.

[25] Comite P, Cobianchi L, Avanzini MA, Zonta S, Mantelli M, Achille V, et al. Isolation and ex vivo expansion of bone marrow-derived porcine mesenchymal stromal cells: potential for application in an experimental model of solid organ transplantation in large animals. Transplantation proceedings. 2010;42:1341-3.

[26] Peterbauer-Scherb A, van Griensven M, Meinl A, Gabriel C, Redl H, Wolbank S. Isolation of pig bone marrow mesenchymal stem cells suitable for one-step procedures in chondrogenic regeneration. Journal of tissue engineering and regenerative medicine. 2010;4:485-90.

[27] Pittenger MF. Mesenchymal stem cells from adult bone marrow. Methods in molecular biology. 2008;449:27-44.

[28] Bronckaers A, Hilkens P, Martens W, Gervois P, Ratajczak J, Struys T, et al. Mesenchymal stem/stromal cells as a pharmacological and therapeutic approach to accelerate angiogenesis. Pharmacology \& therapeutics. 2014;143:181-96.

[29] Ibrahim AG, Cheng K, Marban E. Exosomes as critical agents of cardiac regeneration triggered by cell therapy. Stem cell reports. 2014;2:606-19.

[30] Bian S, Zhang L, Duan L, Wang X, Min Y, Yu H. Extracellular vesicles derived from human bone marrow mesenchymal stem cells promote angiogenesis in a rat myocardial infarction model. Journal of molecular medicine. 2014;92:387-97.

[31] Fan M, Chen W, Liu W, Du GQ, Jiang SL, Tian WC, et al. The effect of age on the efficacy of human mesenchymal stem cell transplantation after a myocardial infarction. Rejuvenation research. 2010;13:429-38. 
[32] Kuraishi K, Iwata H, Nakano S, Kubota S, Tonami H, Toda M, et al. Development of nanofibercovered stents using electrospinning: in vitro and acute phase in vivo experiments. Journal of biomedical materials research Part B, Applied biomaterials. 2009;88:230-9.

[33] Dong Y. Nanofiber Covered Stent for Vascular Diseases [PhD Dissertation]. Singapore: National University of Singapore; 2009.

[34] Kim SY, Kim M, Kim MK, Lee H, Lee DK, Lee DH, et al. Paclitaxel-eluting nanofiber-covered selfexpanding nonvascular stent for palliative chemotherapy of gastrointestinal cancer and its related stenosis. Biomedical microdevices. 2014.

[35] Lee CH, Chang SH, Lin YH, Liu SJ, Wang CJ, Hsu MY, et al. Acceleration of re-endothelialization and inhibition of neointimal formation using hybrid biodegradable nanofibrous rosuvastatin-loaded stents. Biomaterials. 2014;35:4417-27.

[36] Lee CH, Lin YH, Chang SH, Tai CD, Liu SJ, Chu Y, et al. Local sustained delivery of acetylsalicylic acid via hybrid stent with biodegradable nanofibers reduces adhesion of blood cells and promotes reendothelialization of the denuded artery. International journal of nanomedicine. 2014;9:311-26.

[37] Blindt R, Vogt F, Astafieva I, Fach C, Hristov M, Krott N, et al. A novel drug-eluting stent coated with an integrin-binding cyclic Arg-Gly-Asp peptide inhibits neointimal hyperplasia by recruiting endothelial progenitor cells. Journal of the American College of Cardiology. 2006;47:1786-95.

[38] den Dekker WK, Houtgraaf JH, Onuma Y, Benit E, de Winter RJ, Wijns W, et al. Final results of the HEALING IIB trial to evaluate a bio-engineered CD34 antibody coated stent (GenousStent) designed to promote vascular healing by capture of circulating endothelial progenitor cells in CAD patients. Atherosclerosis. 2011;219:245-52.

[39] Kang CK, Lim WH, Kyeong S, Choe WS, Kim HS, Jun BH, et al. Fabrication of biofunctional stents with endothelial progenitor cell specificity for vascular re-endothelialization. Colloids and surfaces B, Biointerfaces. 2013;102:744-51.

[40] Klomp M, Beijk MA, de Winter RJ. Genous endothelial progenitor cell-capturing stent system: a novel stent technology. Expert review of medical devices. 2009;6:365-75.

[41] Silber S, Damman P, Klomp M, Beijk MA, Grisold M, Ribeiro EE, et al. Clinical results after coronary stenting with the Genous Bio-engineered R stent: 12-month outcomes of the e-HEALING (Healthy Endothelial Accelerated Lining Inhibits Neointimal Growth) worldwide registry. Eurolntervention : journal of EuroPCR in collaboration with the Working Group on Interventional Cardiology of the European Society of Cardiology. 2011;6:819-25. 


\section{Figure Captions}

Figure 1: Schematic of the structure of the stem cell impregnated nanofiber stent sleeve. An inner sleeve electrospun directly on the stent provides the primary barrier between stem cells (light blue) and the blood vessel lumen (red). An optional outer sleeve overcoat provides a barrier between stem cells and the blood vessel wall (dark blue). Paracrine factors (yellow) freely pass through the sleeve. Cells, being much larger than the sleeve pore size, do not pass through the sleeve.

Figure 2: Scanning electron micrograph demonstrating the stent uniformly covered with the NF sleeve (A). Average NF sleeve pore diameter was $\sim 1.0$ um at baseline and $~$ 0.9 um after balloon re-expansion of the stent, with a relatively narrow distribution (B). NF sleeve fiber structure electrospun at $12 \mathrm{kV}$ at baseline (C) vs. after balloon reexpansion (D) shows minor stretch artifact. NF sleeve structure at $18 \mathrm{kV}$ shows more uniform fiber orientation $(E)$ without significant changes in pore size.

Figure 3: Confocal imaging of the curved surface of the NF stent sleeves 7 days after seeding with MSCs shows a monolayer of highly confluent cells, with cross-sections showing no cell infiltration into the NF sleeve matrix.

Figure 4: Maximum intensity projections of confocal microscopy images of MSCs on the NF sleeve located between stent struts $(A, C)$ and located close to the stent struts (B, D) show increased viability for cells located on the sleeve between stent struts. 
Viable cells stain green and non-viable cells stain red. Initially sparse MSC coverage on Day $1(A, B)$ increased to near confluence on Day $8(C, D)$. Controls $(E, F)$ are NF stent sleeves not seeded with cells. The white star denotes location of the stent strut.

Figure 5: Overcoating MSC impregnated NF sleeves with an additional NF sleeve layer (A) after 7 days in culture resulted in an initial steep drop in cell count (B), but then nearly complete re-population 6 days later (C). Viable cells stain green, non-viable cells stain red.

Figure 6: Stem cell surface marker expression patterns typical of stem cells were retained in MSCs embedded within the NF stent sleeve. Antibody-specific staining in red and isotype negative control in blue.

Figure 7: Cumulative production of vascular endothelial growth factor (VEGF) and hepatocyte growth factor (HGF) by MSCs on NF sleeves over 7 days.

Figure 8: Induced organization of human umbilical vein endothelial cells (HUVECs) into tubules by NF sleeves with MSCs compared to NF sleeves without MSCs (negative control) and NF sleeves with LSGS (Low Serum Growth Supplement with pre-loaded PFs, positive control). Compared to negative control, HUVEC tubule lengths are $3.1 \pm 0.7$ fold greater $(p<0.01)$ when exposed to NF sleeves with MSCs with no NF overcoat, and $3.6 \pm 0.1$ fold greater $(p<0.01)$ when exposed to NF sleeves with MSCs with a NF overcoat. Remarkably, even though NF sleeves with MSCs are not pre- 
loaded with PFs, enough PFs are generated by the NF sleeve MSCs within 18 hours to match tubule formation of the NF sleeves with LSGS containing pre-loaded PFs. The lower panels show an example of the spontaneous organization of HUVECs (left) into tubules (right) induced by the NF sleeve MSCs.

Figure 9: Angiographic appearance of the pig umbilical artery $(A)$ prior to implantation of a NF stent sleeve impregnated with allogeneic MSCs. After stent sleeve deployment (B), the artery remained fully patent at 60 minutes (C) and at 7 days (D). 
Figure 1

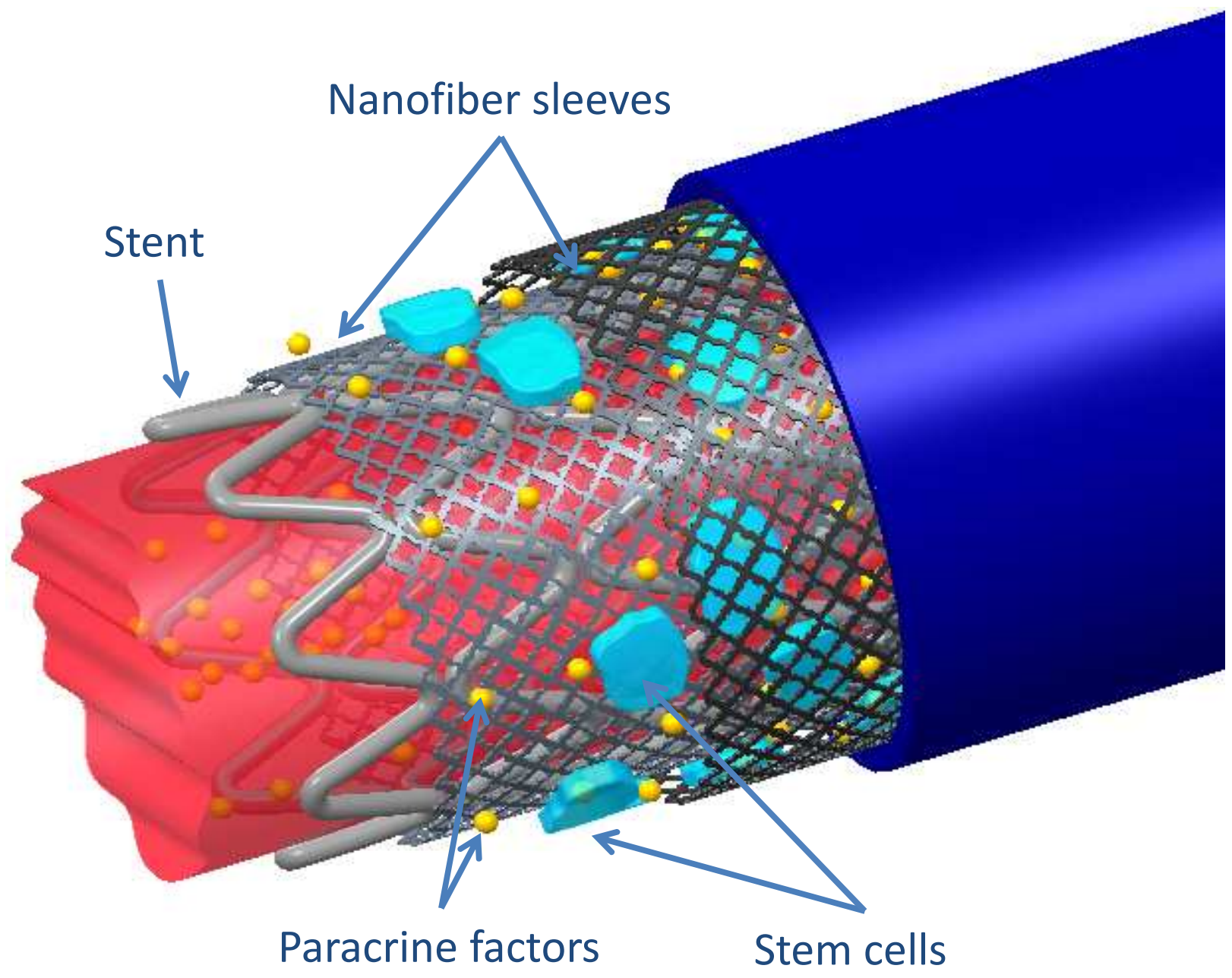


Figure 2
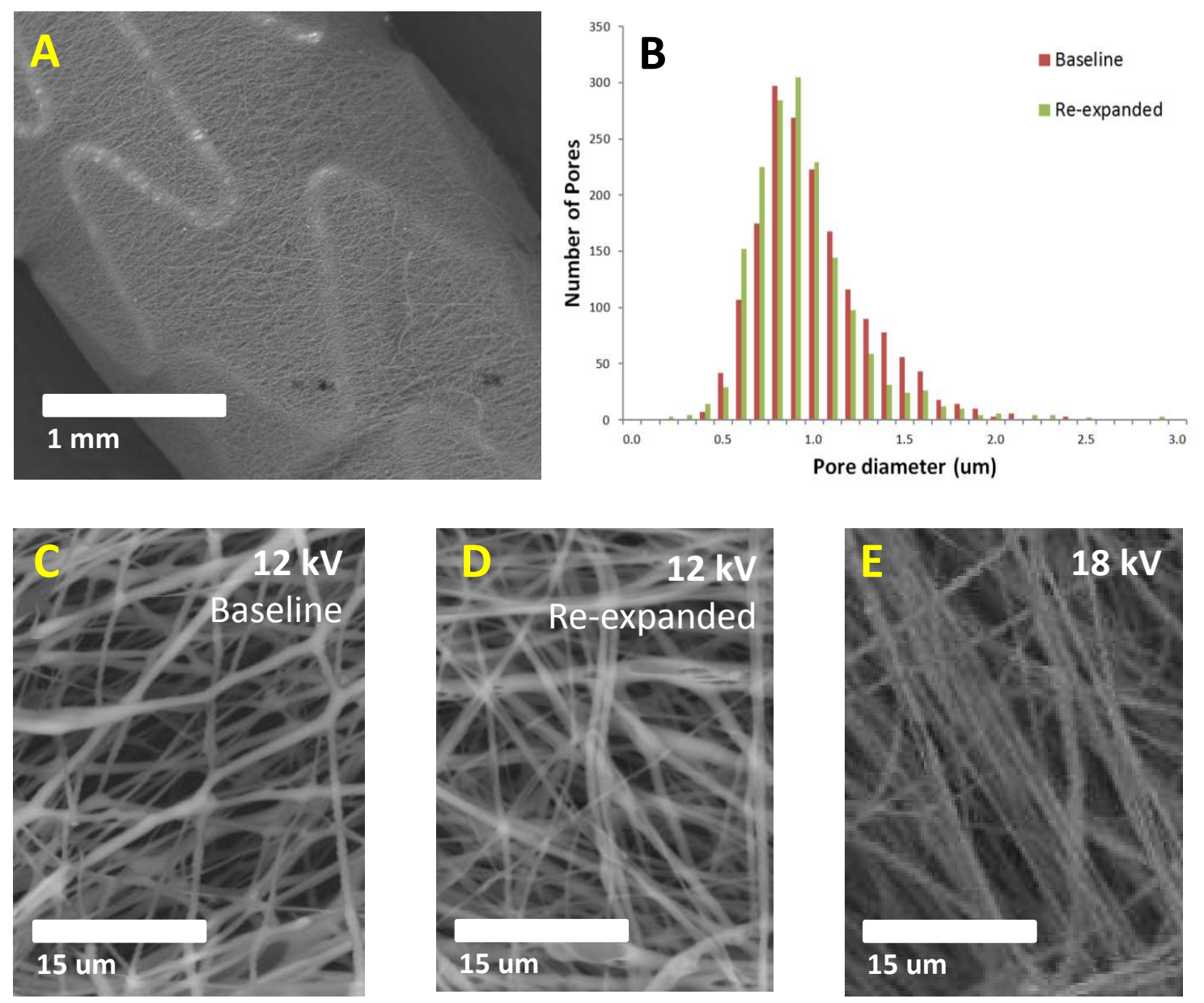
Figure 3

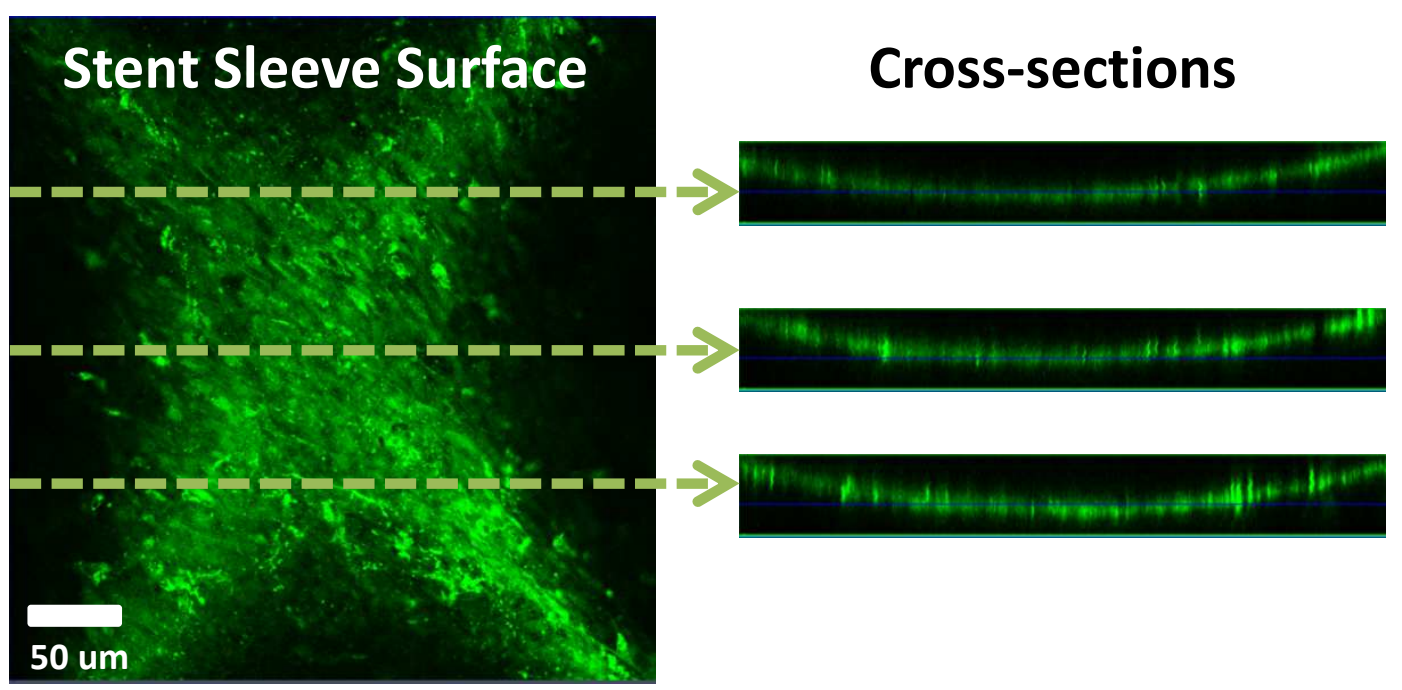


Figure 4

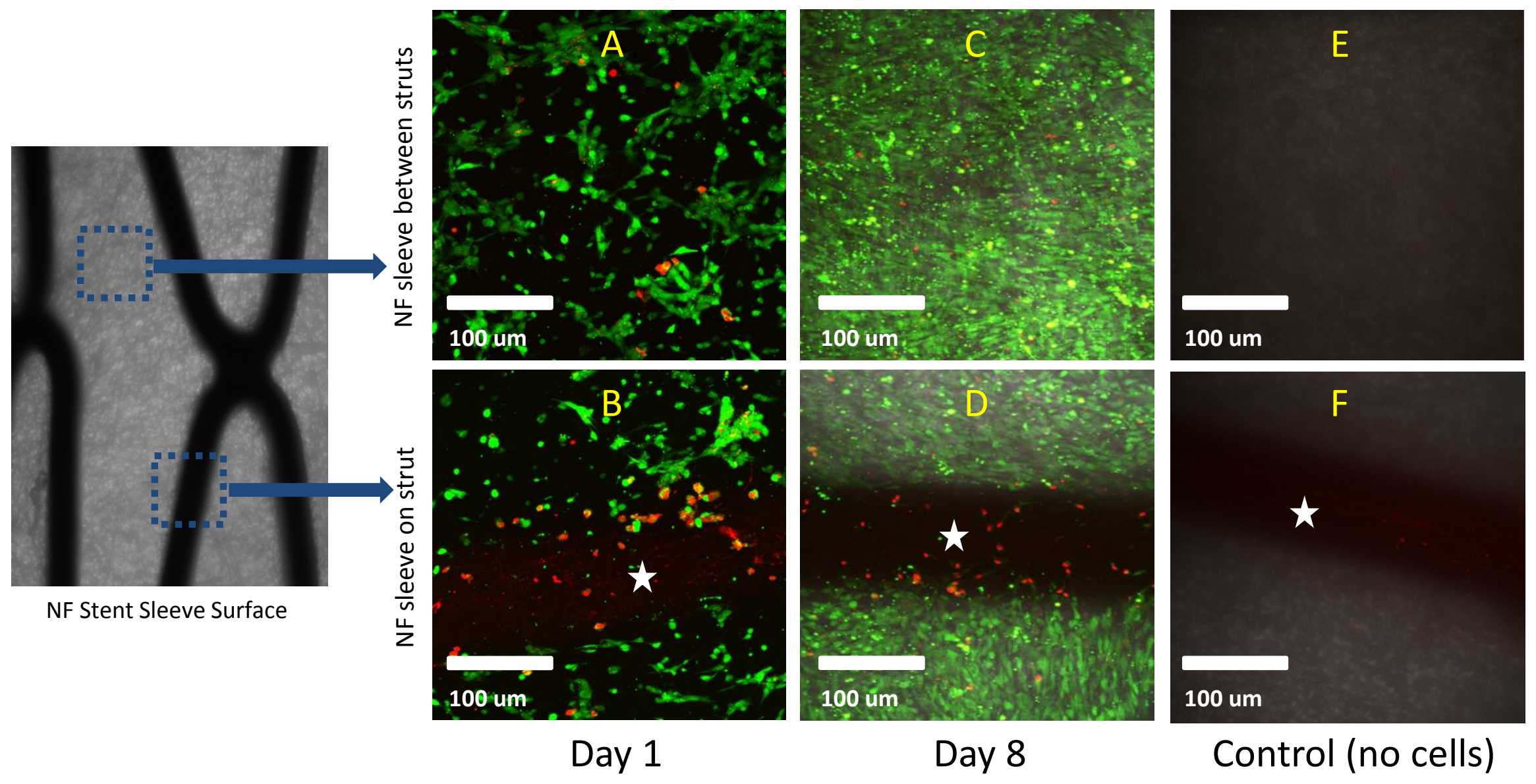


Figure 5

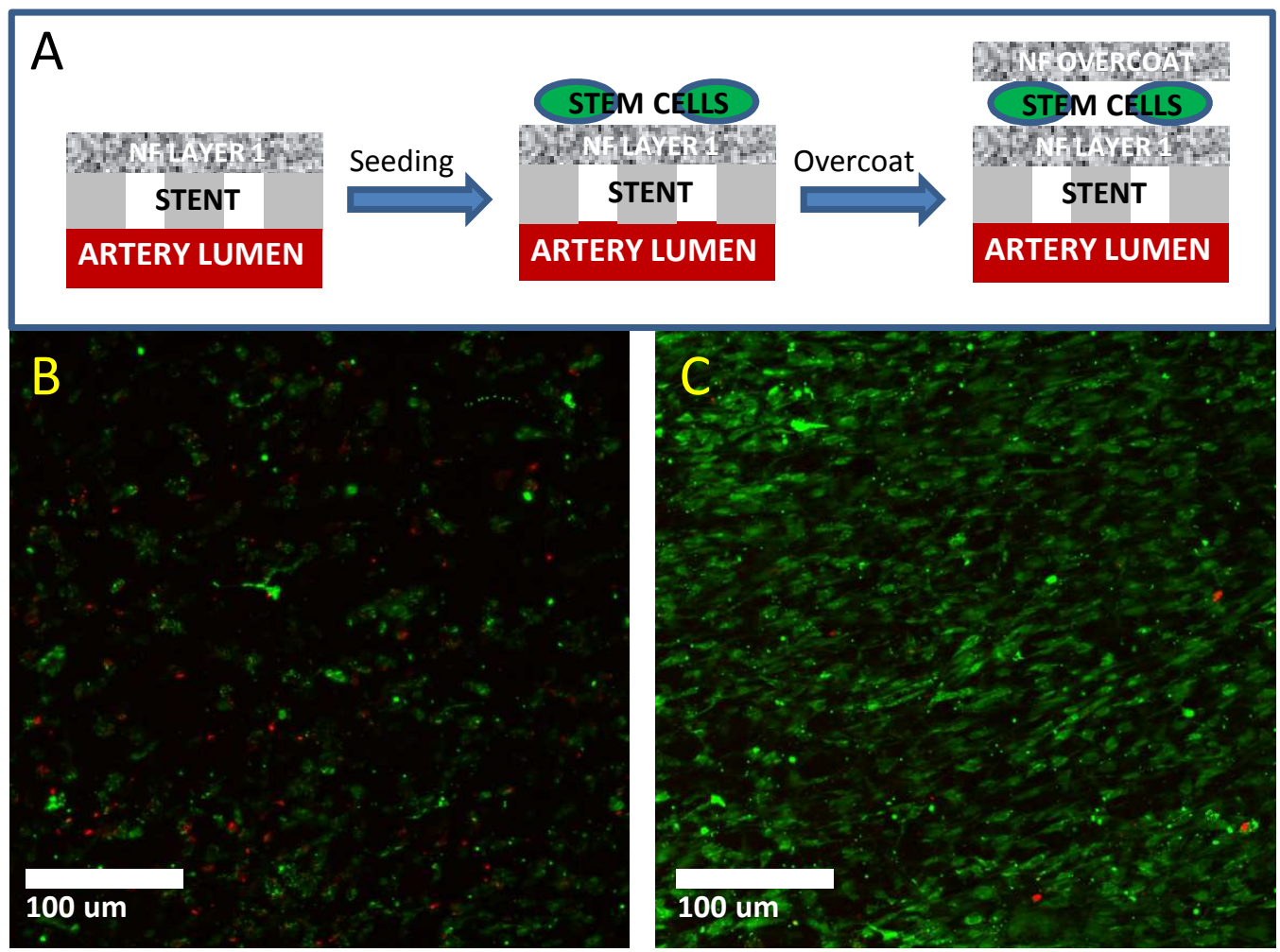


Figure 6
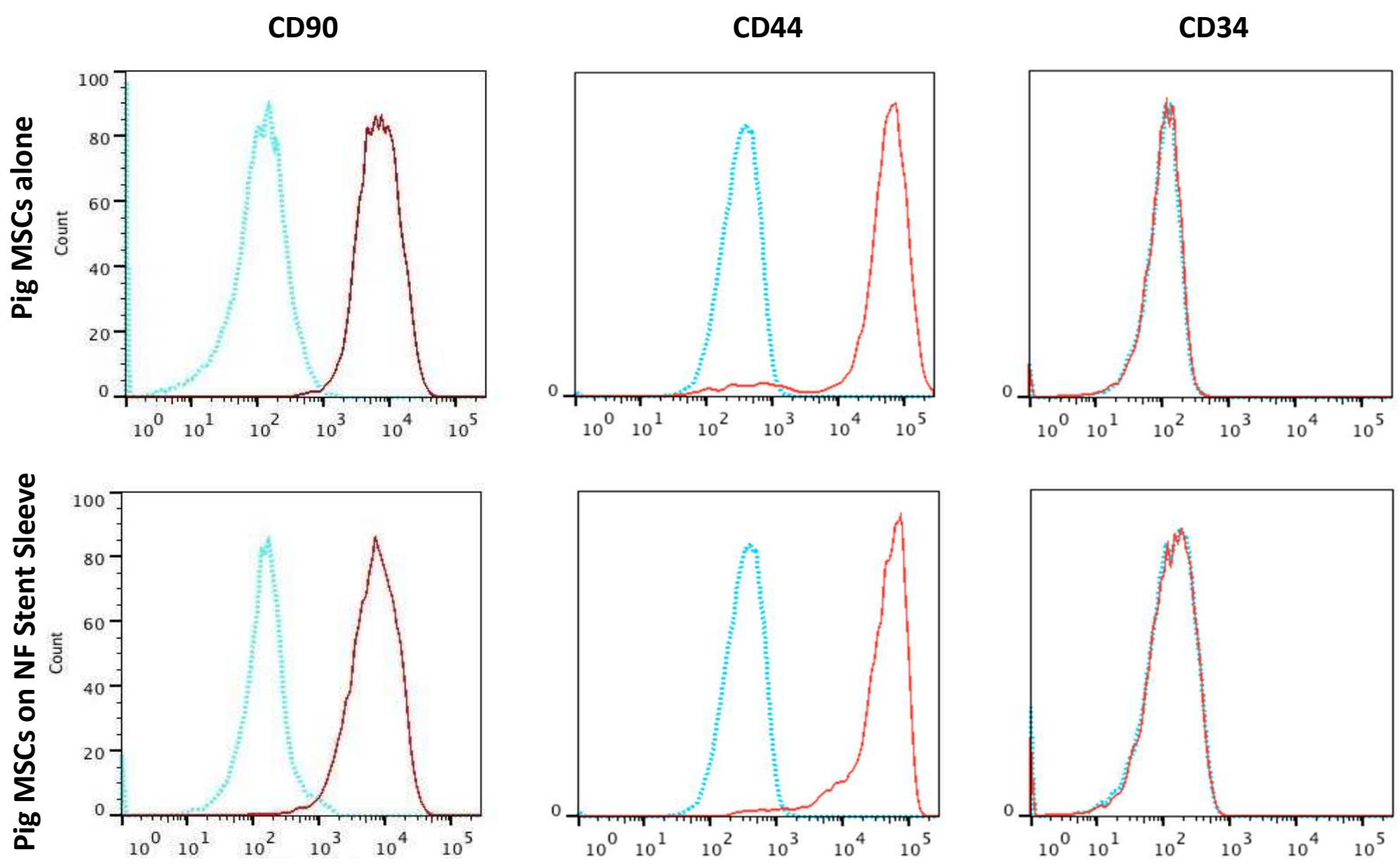
Figure 7
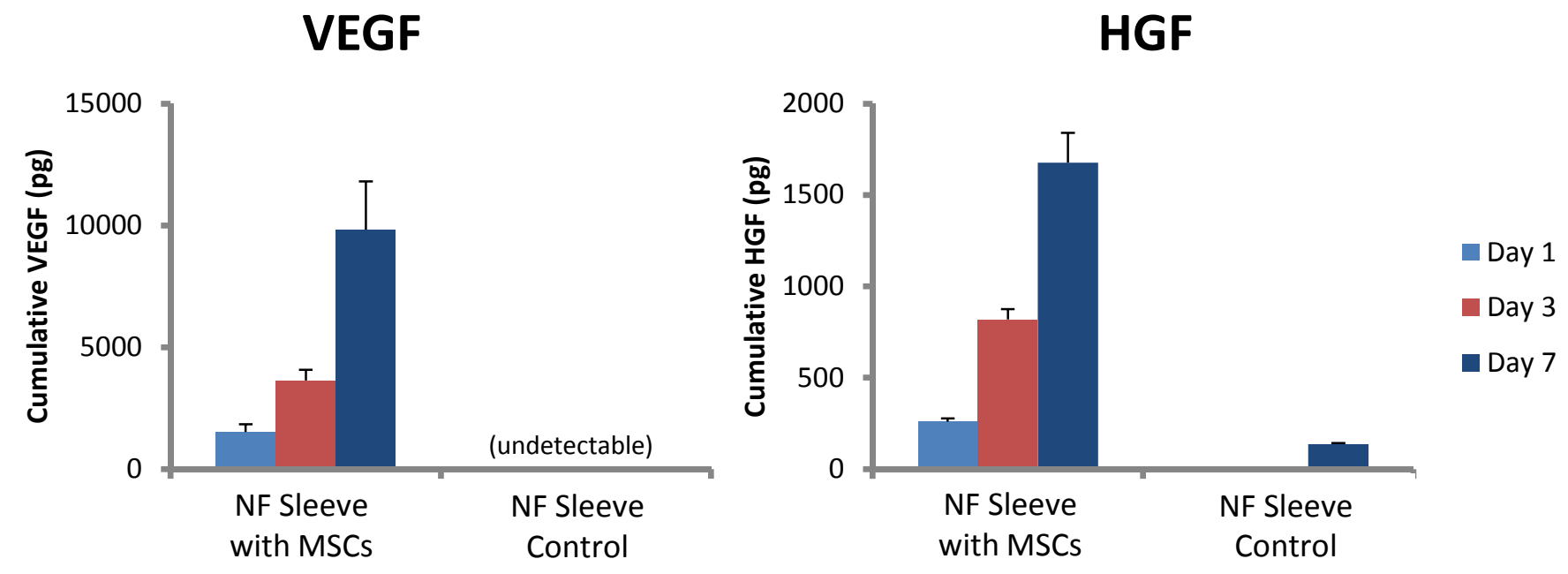
Figure 8
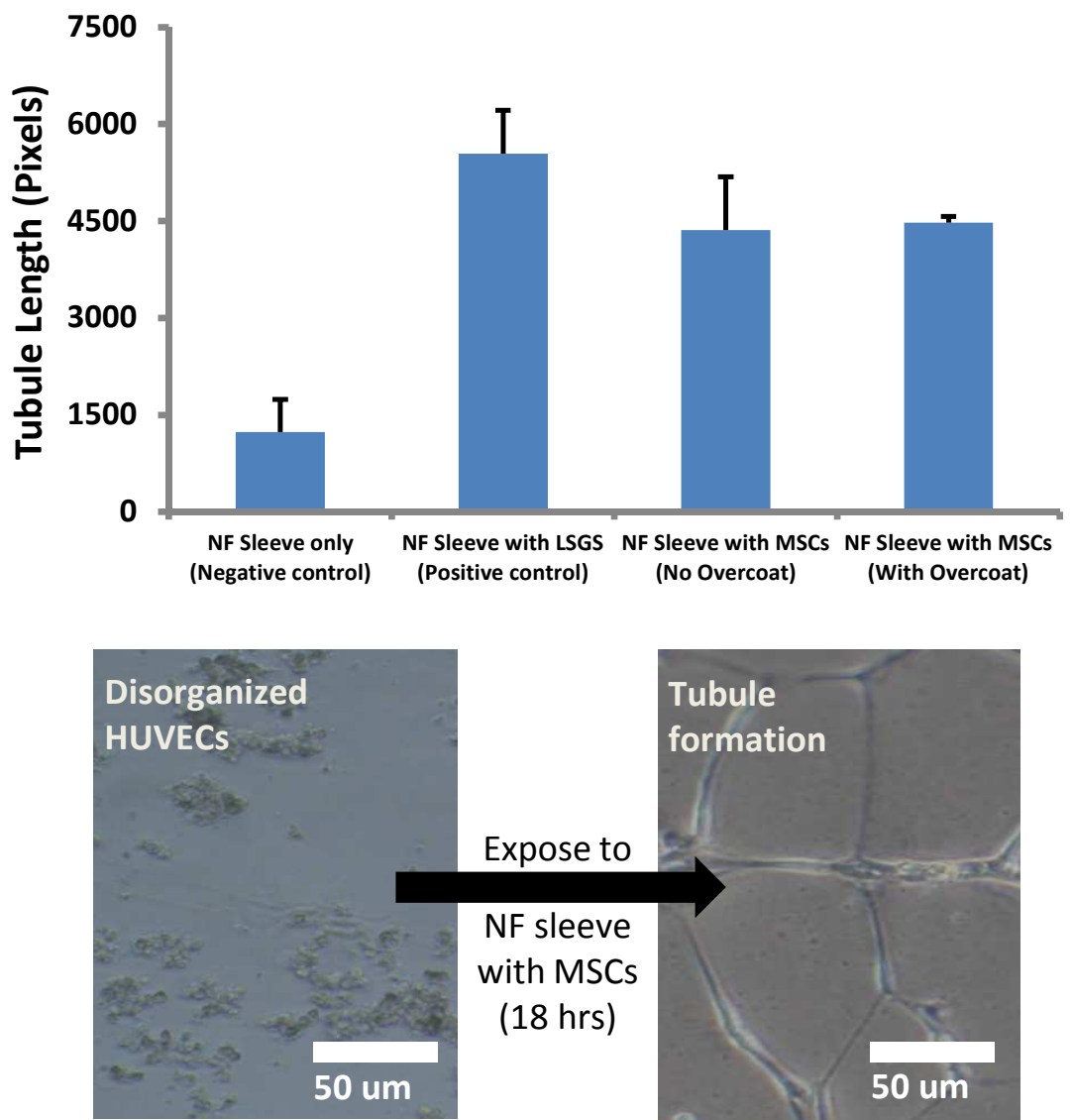


\section{Figure 9}

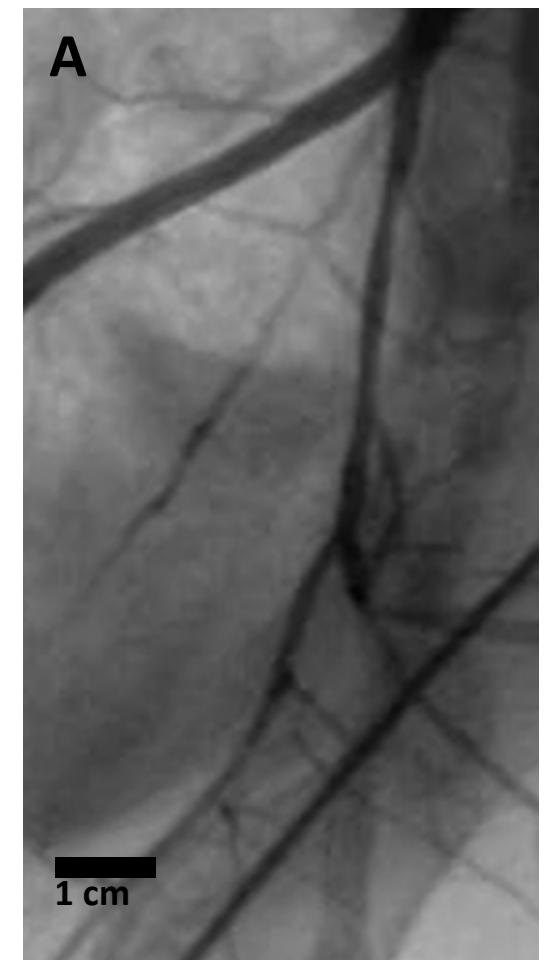

Pre-deployment

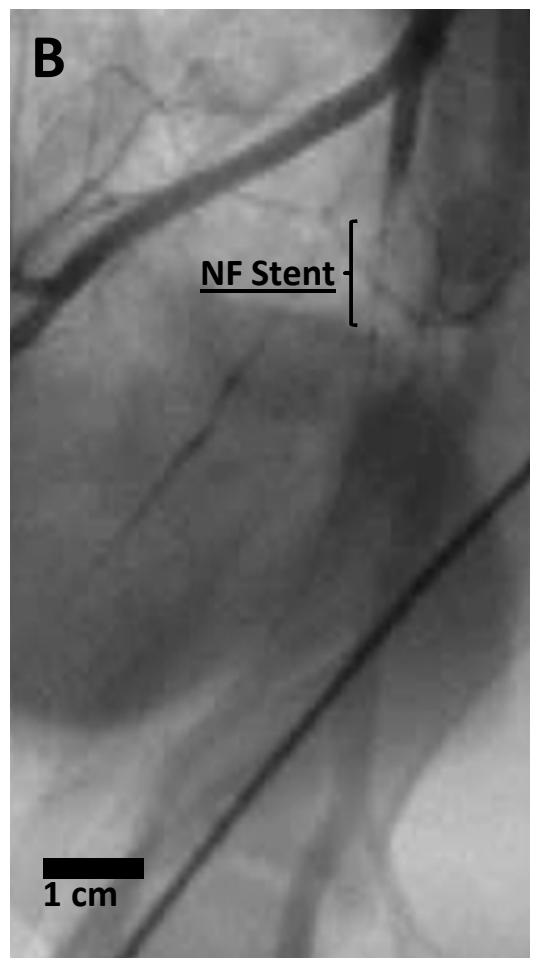

During deployment

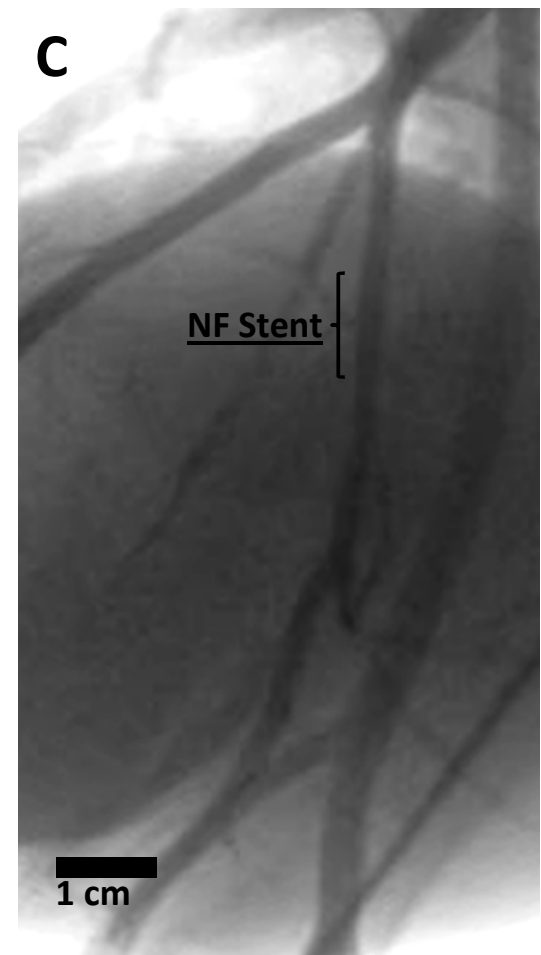

60 min post-deployment

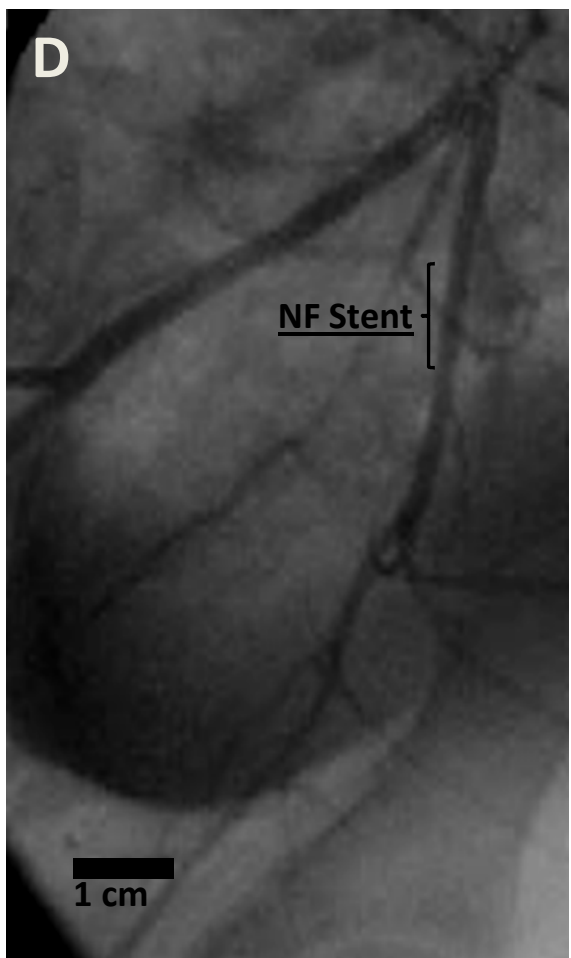

7 days post-deployment 